\title{
Non-Abelian spin singlet states of two-component Bose gases in artificial gauge fields
}

\author{
T. Graß ${ }^{1}$, B. Juliá-Díaz ${ }^{1}$, N. Barberán ${ }^{2}$, and M. Lewenstein ${ }^{1,3}$ \\ ${ }^{1}$ ICFO-Institut de Ciències Fotòniques, Parc Mediterrani de la Tecnologia, 08860 Barcelona, Spain \\ ${ }^{2}$ Dept. ECM, Facultat de Física, U. Barcelona, 08028 Barcelona, Spain and \\ ${ }^{3}$ ICREA-Institució Catalana de Recerca i Estudis Avançats, 08010 Barcelona, Spain
}

\begin{abstract}
We study strongly correlated phases of a pseudo-spin-1/2 Bose gas in an artificial gauge field using the exact diagonalization method. The atoms are confined in two dimensions and interact via a two-body contact potential. In Abelian gauge fields, pseudo-spin singlets are favored by pseudospin independent interactions. We find a series of incompressible phases at fillings $\nu=2 k / 3$. By comparison with the non-Abelian spin singlet (NASS) states, constructed as zero-energy eigenstates of a $(k+1)$-body contact interaction, we classify the non-trivial topology of the states. An additional spin-orbit coupling is shown to switch between NASS-like states and spin-polarized phases from the Read-Rezayi series.
\end{abstract}

PACS numbers: 73.43.-f,03.65.Vf,37.10.Vz

Keywords: Gauge fields. Ultracold Bose gases. Correlated states. Exact diagonalization.

Topological phases of matter attract a great deal of attention since the late 1980s, when the fractional quantum Hall (FQH) effect of electrons in two dimensions (2D) subjected to a strong perpendicular magnetic field was recognized as a paradigm of such phases [1]. It was then pointed out that amongst these states there might be some which support non-Abelian excitations, where the exchange of two quasiparticles is described by some unitary matrix, as it shifts between different degenerate ground states [2]. Recently, this property has triggered the interest in topological phases, as quantum gates operating with non-Abelian anyons might pave the way to fault-tolerant quantum computers [3]. For this purpose, the possibility of controlling or even designing quantum many-body states seems to be advantageous if not inevitable. A very important tool to do so are cold atoms which can, in various ways, be manipulated through interactions with light [4]. Despite being electroneutral, the atoms may even couple to external gauge potentials, which, instead of being true electromagnetic vector potentials, have to be synthesized. Artificial magnetic fields have been achieved experimentally by rotating the atomic cloud [5] (see also [6] for a review), though instabilities at high rotation frequencies have so far prevented from reaching the highly correlated regime. A promising alternative pursues the generation of gauge fields by a laser dressing of the atoms (7). This method has already been proven experimentally 8 . It is readily generalized to non-Abelian gauge fields [9], and has recently allowed for synthesizing a spin-orbit coupling [10].

The strongly correlated states occurring in such systems have been numerically investigated for the case of spinless or spin-polarized bosons [11], in which the incompressible phases are well described by the Read-Rezayi (RR) series of parafermionic states [12], including the Laughlin wave function [13], and the Moore-Read (MR) wave function [2]. Although also bosons with a pseudospin-1/2 degree of freedom have already been condensed
14, and their behavior under rotation has been studied both theoretically [15] and experimentally [16], few attention has yet been paid to the strongly correlated regime in such systems. For the electronic FQH effect, however, a variety of states have been proposed for systems with spin, which despite the strong magnetic field might be in a singlet phase due to the small gyromagnetic ratio of electrons in many solids. Within a conformal field theory (CFT) framework, a generalization of the RR series to the so-called non-Abelian spin singlet (NASS) series 17] has been formulated. Here the term "non-Abelian" refers to the nature of the excitations. For bosons, this series of topological states occurs at fillings $\nu=2 k / 3$, and includes the Halperin state [18] with Abelian excitations at $k=1$. Like the RR states, the NASS states are constructed as the zero-energy eigenstates of a repulsive $(k+1)$-body contact interaction.

In this paper we show, using exact diagonalization, that also two-body contact (2BC) interaction leads to the formation of incompressible states precisely at the NASS filling factors in a 2D Bose gas exposed to an Abelian gauge field. For $k=1$, this is trivially the Halperin state, but also for $k=2$ significant overlap with the corresponding NASS state and a similar spectral structure indicate that the system can be brought into the NASS phase within this realistic setup.

This finding is in close analogy to the spin-polarized case with RR-like states [11] at filling $\nu=k / 2$. However, as it is experimentally difficult to get into the regime of low filling, the two-component system benefits from supporting analogs of the RR states at higher filling. Furthermore, its possibility of tuning independently interspin and intra-spin interactions provides additional control. For pseudo-spin-independent repulsive 2BC interactions, a mechanism is discussed which favors pseudo-spin singlet states. We show that, counterintuitively, states with zero interspin interaction energy are obtained by increasing the interspin interaction strength. Finally, ef- 
fects stemming from a spin-orbit ( $\mathrm{SO}$ ) coupling can be integrated in the framework of two-component gases by generalizing to a non-Abelian gauge field. We investigate this scenario, and find a transition from the singlecomponent RR states [19, 20] to the NASS states at SO coupling strengths corresponding to a Landau level (LL) degeneracy.

We consider a two-component Bose gas in 2D, which on the single-particle level is described by the Hamiltonian $H_{\mathrm{sp}}=(\boldsymbol{p}-\boldsymbol{A})^{2} /(2 m)$. The vector potential $\boldsymbol{A}$ is given by

$$
\boldsymbol{A}=B(0, x, 0) \mathbb{1}+q\left(\sigma_{x}, \sigma_{y}, 0\right)
$$

The first term, acting equally on both pseudo-spin components, describes a magnetic field of strength $B$ in the Landau gauge. The second term, with $\sigma_{i}$ being the Pauli matrices, accounts for a Rashba SO coupling controlled by the coupling strength $q$, which we set to zero in the first part of this work. A detailed description how to implement this Hamiltonian for electroneutral atoms can be found in the supplementary material of Ref. [19]. As the boundary conditions we choose a periodic square of length $a$. This eliminates edge effects from our study, and, moreover, generates a non-trivial toroidal geometry, on which topological phases can be classified by the number of degenerate ground states [1]. Solving the singleparticle Hamiltonian yields, for $q=0$, the usual LL structure [21], where every state $|n, j, s\rangle$ within a fully degenerate Landau level $n$ is characterized by a momentum quantum number $j$ and an additional two-fold degenerate spin quantum number $s=\uparrow, \downarrow$. Since an energy gap $2 B$ separates the different LLs, we restrict ourselves to the lowest LL, $n=0$. Fixing the number of fluxes per unit cell, $N_{\Phi}$, restricts $j$ to values running from 1 to $N_{\Phi}$, and the many-body Hamiltonian is, up to a constant amounting for the kinetic energy, given by

$$
\hat{\mathrm{H}}=\sum_{\{j, s\}} V_{\{j, s\}} \hat{\mathrm{a}}_{j_{1} s_{1}}^{\dagger} \hat{\mathrm{a}}_{j_{2} s_{2}}^{\dagger} \hat{\mathrm{a}}_{j_{3} s_{3}} \hat{\mathrm{a}}_{j_{4} s_{4}}
$$

where $\hat{a}_{j s}$ annihilates a particle in state $|0, j, s\rangle$, and $\{j, s\}$ denotes the set of quantum numbers $j_{1}, \ldots, j_{4}$ and $s_{1}, \ldots, s_{4}$. The interaction conserves total momentum $J=\sum_{i} j_{i} \bmod N_{\Phi}$, and the spin of the particles, so the coefficients read

$$
\begin{aligned}
V_{\{j, s\}}= & \delta_{j_{1}+j_{2}, j_{3}+j_{4}}^{\prime} \delta_{s_{1}, s_{3}} \delta_{s_{2}, s_{4}} \times \\
& \left\langle j_{1}, s_{1}\left|\otimes\left\langle j_{2}, s_{2}\left|g_{s_{1} s_{2}} \delta\left(z_{1}-z_{2}\right)\right| j_{3}, s_{3}\right\rangle \otimes\right| j_{4}, s_{4}\right\rangle,
\end{aligned}
$$

with $g_{s_{1} s_{2}}$ the spin-dependent contact interaction strength of two particles, and $\delta^{\prime}$ a Kronecker delta modulo $N_{\Phi}$. The Hamiltonian is diagonalized in blocks with a fixed particle number $N$, defining the filling factor $\nu=N / N_{\Phi}$, fixed Haldane momentum $\boldsymbol{K}[22$ ], and a fixed spin polarization $S=N_{\uparrow}-N_{\downarrow}$, where $N_{\uparrow}\left(N_{\downarrow}\right)$ denotes the number of spin-up (spin-down) particles.
First, we show that for spin-independent interactions, i.e. $g_{s_{1} s_{2}}=g$, the singlet state is energetically favored. This configuration is a natural choice, as many experiments 14] work with the $\left|F=1, m_{F}=0,1\right\rangle$ states of ${ }^{23} \mathrm{Na}$, or the $\left|F=1, m_{F}=-1\right\rangle$ and $\left|F=2, m_{F}=1\right\rangle$ states of ${ }^{87} \mathrm{Rb}$ with almost equal s-wave scattering lengths within and between the components. We start by contrasting the singlet to the fully polarized situation, $S=N$, in which we recover the results from Ref. [11], with incompressible phases at $\nu=k / 2$, forming the RR series. The fully polarized zero-energy state with highest filling has $\nu=1 / 2$, and is the Laughlin state. In the opposite limit of a fully unpolarized system, zero-energy states occur up to $\nu=2 / 3$, where the 221-Halperin state is the exact unique ground state of the $2 \mathrm{BC}$ interaction. The analytic expression of this state on a disc reads

$$
\Psi_{\mathrm{H}}=\prod_{i<j}^{N_{\uparrow}}\left(z_{i \uparrow}-z_{j \uparrow}\right)^{2} \prod_{k<l}^{N_{\downarrow}}\left(z_{k \downarrow}-z_{l \downarrow}\right)^{2} \prod_{i, k}\left(z_{i \uparrow}-z_{k \downarrow}\right),(4)
$$

where we omit the irrelevant exponential term $\exp \left(-\sum\left|z_{i}\right|^{2} / 4\right)$. This wave function vanishes whenever two particles are at the same position, and it is symmetric under exchange of two spin-up or two spin-down particles. However, it is antisymmetric under exchange of particles with different spin, and thus cannot be used for describing spinless bosons. For this case, also the terms in the last product had to be squared, which would result in a less dense Laughlin-like wave function. Generalizing this observation, we state that any spatial wave function which solves the fully polarized problem must also be a solution for $|S|<N$, while the opposite is not true. Thus, for a spin-independent interaction, it strictly follows for the ground state energies $E\left(S_{i}\right)$ with $\left|S_{1}\right|<\left|S_{2}\right|$ that $E\left(S_{1}\right) \leq E\left(S_{2}\right)$. Our numerical data, partially shown in the left side of Fig. 11 supports this finding. In fact, in all cases we have investigated, the ground state of the $S=2$ subspace occurs in the spectrum of $S=0$ as a lowlying excitation, except for $N=6$ and $N_{\Phi}=8$, where we have a degeneracy, $E(S=0)=E(S=2)$. In most cases, thus, the ground state is a singlet, which is understood by noting that interactions of a $\uparrow \downarrow$-pair can be fully avoided by a single zero of the form $z_{i \uparrow}-z_{k \downarrow}$ in the wave function, while at least two zeros must be spent if a pair of particles with equal spin is to be anticorrelated in a similar way.

Feshbach resonances allow to tune the spindependency of interactions (cf. [4]). Increasing $g_{\uparrow \downarrow}$ while leaving $g_{\uparrow \uparrow}=g_{\downarrow \downarrow}=g$ should favor spin polarizations with less $\uparrow \downarrow$-pairs. However, this does not necessarily drive the system into the fully polarized configuration: As shown in Fig. 2 for $N=6$ at $\nu=1$, the energy of even the fully unpolarized system saturates for large $g_{\uparrow \downarrow}$. In this limit, the real ground state has $S=2$ and $E=0.449$ (in units of $g B$ ), close to the $S=0$ and $S=4$ ground states with $E=0.466$ and $E=0.475$. 


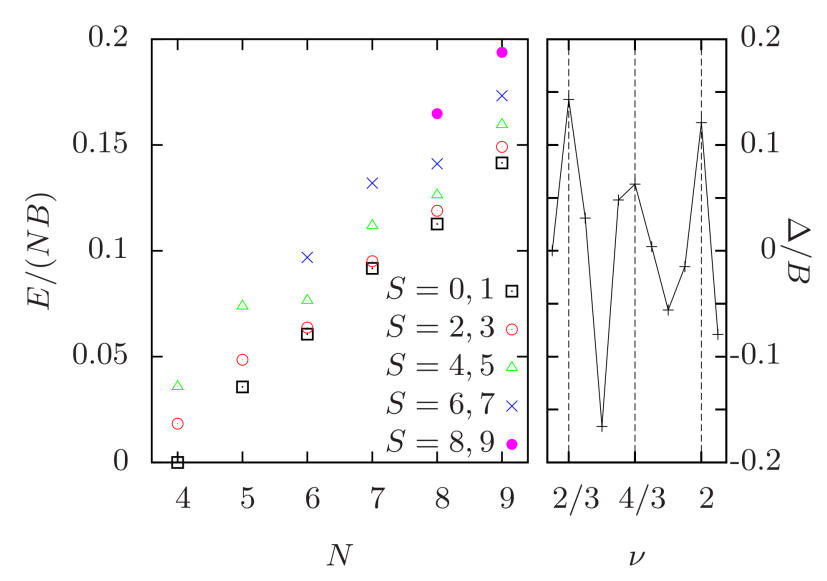

FIG. 1. (Color online) Left: Ground state energies, not showing possible degeneracies, at $N_{\Phi}=6$ for different $N$ and $S$ subspaces with $g_{s_{1} s_{2}}=g$. Even (odd) values of $S$ correspond to even (odd) $N$. Right: $\Delta$ vs. $\nu$ for $N_{\Phi}=6$ and $g_{s_{1} s_{2}}=g$.
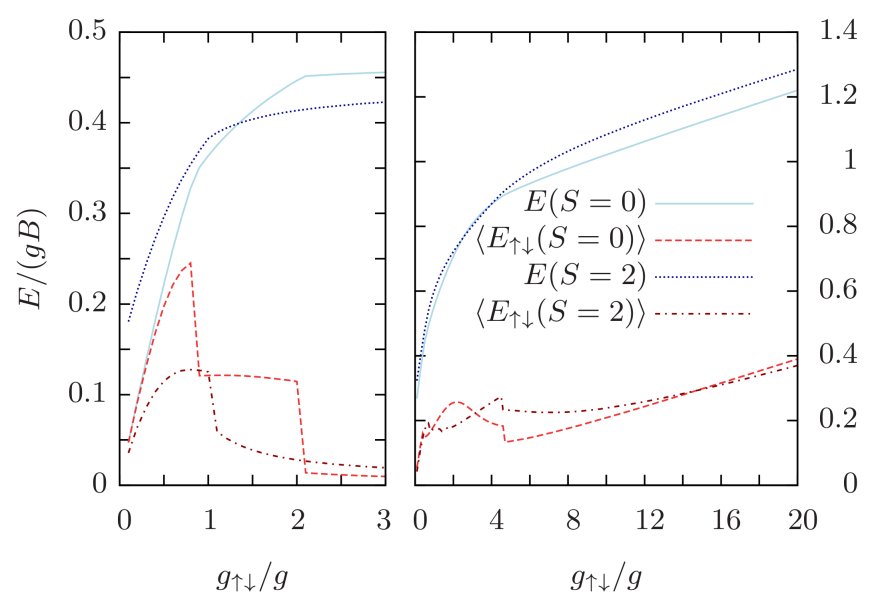

FIG. 2. (Color online) Interaction energy for $N=6$ and $S=0,2$ as a function of $g_{\uparrow \downarrow}$. Left: $N_{\Phi}=6$. Right: $N_{\Phi}=5$.

All of them are well separated from the fully-polarized ground state with $E=0.581$. In contrast to this, the energy of spin-unpolarized systems will never saturate at larger filling, as is shown in Fig. 2 for $\nu=6 / 5$. This different behavior is understood by noting that for $\nu=1$ the number of available zeros in the wave function is sufficient to completely suppress interactions between pairs of different spins, $\left\langle E_{\uparrow \downarrow}\right\rangle \rightarrow 0$, while it is not for larger $\nu$. Jumps in the curve of $\left\langle E_{\uparrow \downarrow}\right\rangle$ as a function of $g_{\uparrow \downarrow}$ show that the states with $\left\langle E_{\uparrow \downarrow}\right\rangle=0$ are reached by several abrupt re-organizations of the ground states. We note that once the state with $\left\langle E_{\uparrow \downarrow}\right\rangle=0$ is obtained, this state must be an eigenstate for arbitrary $g_{\uparrow \downarrow}$. Thus, by abruptly switching off the interspin interaction, one could produce binary mixtures of highly entangled, non-interacting systems.

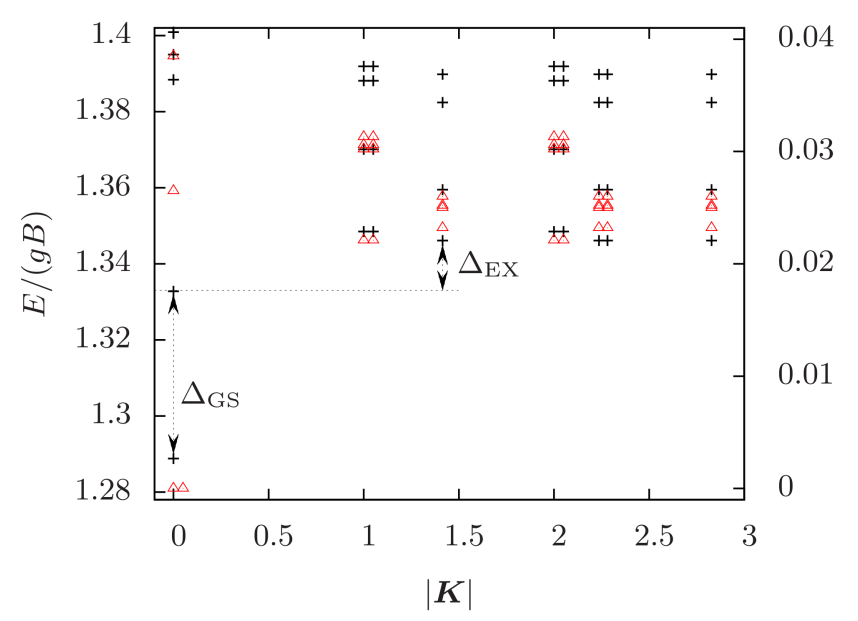

FIG. 3. (Color online) Energy spectra of singlet states for $N=12$ particles at filling $\nu=4 / 3$. Black crosses (with the left energy scale) show the results for an $\mathrm{SU}(2)$-symmetric $2 \mathrm{BC}$ interaction, while red triangles (with the right energy scale) are the NASS spectrum for any three-body interaction with $g_{s_{1} s_{2} s_{3}}>0$. We do not show the three-fold center-ofmass degeneracy, but visualize further degeneracies by slight shifts in $\boldsymbol{K}$.

Coming back to spin-independent interactions, we ask now the question whether, in analogy to the RR states for spin polarized systems, incompressible states can be found amongst the spin-unpolarized ground states. Since incompressibility is connected to a discontinuity in the chemical potential, we define the particle-hole excitation gap as $\Delta(N)=N[E(N+1) /(N+1)+E(N-1) /(N-$ 1) $-2 E(N) / N]$, which is plotted for $N_{\Phi}=6$ in Fig. 1 (right). Upwards peaks correspond to downward cusps in the energy as a function of $\nu$, and signal incompressible phases. They occur at fillings $\nu=2 k / 3$ with $k \in \mathbb{N}$. For these fillings, a series of singlet states being exact zeroenergy eigenstates of a $(k+1)$-body contact interaction is known [17]. For $k=1$, this is the 221-Halperin state, which has Abelian excitations, while the states for $k>$ 1 are predicted to have non-Abelian excitations, which established the name NASS series.

We first construct these states for $k=2$ and 3 by diagonalizing the three- and four-body contact interaction for up to $N=12$ particles: As predicted by CFT, these states are characterized by a $(k+1)(k+2) / 2$-fold topological degeneracy on the torus. A subtlety occurs at $k=3$, where the CFT construction [17], being restricted to $N=2 k p$ with $p \in \mathbb{N}$, is not able to predict zero-energy ground states for all $N$ possible at $\nu=2$. In fact, we find zero-energy states also for $N=8$ and 10, but, in contrast to the states with $N=6$ and 12 which have the predicted ten-fold degeneracy, they are non-degenerate, and thus belong to a different topological phase.

The overlaps of these states with the ground states of Eq. (2), given in Table [, turn out to be significant: For 


\begin{tabular}{|c|c|c|c|c|c|c|c|}
\hline$N, N_{\Phi}$ & $K$ & 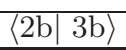 & $E_{2 \mathrm{~b}}$ & $N, N_{\Phi}$ & $K$ & \begin{tabular}{|l|l|}
$\langle 2 \mathrm{~b}\rangle$ \\
\end{tabular} & $E_{2 \mathrm{~b}}$ \\
\hline \multirow[t]{2}{*}{4,3} & $(0,0)$ & 1 & 0.435 & \begin{tabular}{|l|}
12,6 \\
\end{tabular} & $(0,0)$ & 0.334 & 2.604 \\
\hline & & 1 & 0.520 & & & 0.828 & 2.677 \\
\hline \multirow[t]{2}{*}{8,6} & $(0,0)$ & 0.906 & 0.901 & & $(0,2)$ & 0.775 & 2.708 \\
\hline & & 0.920 & 0.908 & & $(0,4)$ & 0.775 & 2.708 \\
\hline \multirow[t]{6}{*}{12,9} & $(0,0)$ & 0.729 & 1.289 & & $(2,0)$ & 0.775 & 2.708 \\
\hline & & 0.885 & 1.333 & & $(2,2)$ & 0.646 & 2.685 \\
\hline & & & & & $(2,4)$ & 0.646 & 2.285 \\
\hline & & & & & $(4,0)$ & 0.775 & 2.708 \\
\hline & & & & & $(4,2)$ & 0.646 & 2.685 \\
\hline & & & & & $(4,4)$ & 0.646 & 2.685 \\
\hline
\end{tabular}

TABLE I. Overlaps between zero-energy eigenstates of $(k+1)$ body interaction with corresponding eigenstates of the $2 \mathrm{BC}$ interaction at energy $E_{2 \mathrm{~b}}$ (in units of $g B$ ) and Haldane momentum $\boldsymbol{K}$ (in units of $2 \pi \hbar / a$ ). All states at filling $\nu=4 / 3$ have an additional three-fold center-of-mass degeneracy.

$k=2$, the states are exactly equal for $N=4$, and even for a relatively large-sized system with $N=12$ the overlaps are still around 0.8. Such a decrease of the overlap with large $N$ also occurs in the case of the RR states in single-component systems with $2 \mathrm{BC}$ interaction [6], suggesting the use of ground state degeneracies to characterize the topological phase in the thermodynamic limit [1]. Topological degeneracies are usually lifted in finite systems, as also happens here. In the case of $\nu=4 / 3$ we may consider the fact that the six lowest eigenstates of $\hat{H}$ agree in their Haldane momenta $\boldsymbol{K}$ with the ones of the sixfold degenerate three-body eigenstates as a signal for belonging to the same topological phase. Both spectra are shown for $N=12$ in Fig. 3. We note that both the degeneracy splitting $\Delta_{\mathrm{GS}}$ and the gap to the excited band $\Delta_{\text {EX }}$ behave non-monotonic with $N$. The situation is different for $\nu=2$, where the candidates for the degenerate ground state manifold mix up with excited states.

Our results therefore suggests that only the $\nu=4 / 3$ NASS phase can be produced with $2 \mathrm{BC}$ interaction. For the experimental feasibility of this state to be high, it should also be robust with respect to deviations from the $\mathrm{SU}(2)$-symmetric interaction. In the case of a $(k+1)$ body contact interaction at $\nu=2 k / 3$, the numerical values of each interaction parameter do not influence the ground states. Though this is different for $2 \mathrm{BC}$ interaction at $\nu=4 / 3$, the overlaps with the NASS states remain high within a wide range of $g_{\uparrow \downarrow} / g$, and have a maximum in the $\mathrm{SU}(2)$-symmetric configuration. For $N=8$, all overlaps are above 0.8 if $g_{\uparrow \downarrow} / g \in[0.8,1.6]$.

Finally, we consider an additional SO coupling with $q>0$ in Eq. (11). This coupling mixes internal and external degrees of freedom on the single-particle level, such that the LLs become superpositions of the form $|n, j, \pm\rangle \equiv \alpha_{n}^{ \pm}|n-1, j, \uparrow\rangle+\beta_{n}^{ \pm}|n, j, \downarrow\rangle$ where $\alpha_{n}^{ \pm}$and $\beta_{n}^{ \pm}$ are functions of $q$ 19. For general $q$, this lifts the previous spin-degeneracy, as the single-particle energies now read $E_{n}^{ \pm}=2 B n+2 q^{2} \pm \sqrt{B^{2}+8 B q^{2} n}$. As has been pointed out in Refs. [19, 20], the system then behaves basically like a single-component system, and the incompressible states are described by the RR series projected into the lowest LL of the SO coupled system. However, for $q^{2} / B=2 n+1$, a degeneracy between $E_{n}^{-}$and $E_{n+1}^{-}$ occurs as the single-particle ground state switches from $|n, j,-\rangle$ to $|n+1, j,-\rangle$. The Hamiltonian from Eq. (2) can then be adapted to the system with SO coupling by identifying the $s$ quantum number with the two-fold degeneracy between $n$ and $n+1$, and by re-defining $V_{\{j s\}}$ accordingly. Note that in this case $S$ does not correspond to the spin polarization, but quantifies the population imbalance between $|n, j,-\rangle$ and $|n+1, j,-\rangle$.

As observed in Ref. [20] within a lowest LL approximation, the projected RR states do not describe the system close to these degeneracy points. For $q^{2} / B=3$, the system becomes gapless at both the Laughlin and the MR filling $\nu=1 / 2$ and 1 . However, we find a clear gap in the spectrum at $\nu=2 / 3$ with $N=6$, and a small gap at $\nu=4 / 3$ with $N=8$. Projecting this $\nu=2 / 3$ state into the lowest LL of the system without SO coupling, and comparing with the 221-Halperin state, we find an overlap of only 0.19 . It should be noted that, in contrast to the previous case of a pure spin degeneracy, interactions now can induce flips within the two-fold degenerate manifold, and therefore $S$ is not conserved. For a complete description of the state, we use generalizations of the Halperin state, obtained by diagonalizing the $2 \mathrm{BC}$ interaction at $\nu=2 / 3$ in an Abelian field $(q=0)$ within different spin subspaces. Then the highest overlap of 0.60 is obtained with the state having $S=-6$, which corresponds to all particles being in $|0, j,-\rangle$. This is also the most populated LL, showing that, despite the degeneracy on the single-particle level, interactions favor the LL with lower $n$. The total fidelity for $-6 \leq S \leq 6$ is 0.82 . For $\nu=4 / 3$ with $N=8$, but now comparing the nondegenerate ground state of the SO coupled system with the ground states of a three-body contact interaction at $q=0$ for different $S$, we still obtain a total fidelity of 0.52 , summing from $-8 \leq S \leq 8$.

We have investigated two-component Bose gases in artificial gauge fields. The possibility of tuning the interaction constants allows to control and manipulate the strongly correlated nature of the eigenstates. Our main finding is the formation of incompressible phases described by the NASS series within the experimentally feasible setup of particles interacting via two-body contact interaction. The internal degree of freedom permits also to realize systems with spin-orbit coupling.

This work has been supported by EU (NAMEQUAM, AQUTE), ERC (QUAGATUA), Spanish MINCIN (FIS2008-00784, FIS2010-16185, FIS2008-01661, 2009 SGR1289), Alexander von Humboldt Stiftung, and AAIIHubbard. B. J.-D. is supported by the Ramón y Cajal program. M. L. acknowledges Hamburg Theory Award.

Note added: After submission of this article, a preprint 
by S. Furukawa and M. Ueda appeared presenting additional numerical evidences for the NASS phase [23].

[1] X. G. Wen and Q. Niu, Phys. Rev. B 41, 9377 (1990)

[2] G. Moore and N. Read, Nucl. Phys. B 360, 362 (1991)

[3] C. Nayak et al., Rev. Mod. Phys. 80, 1083 (2008)

[4] M. Lewenstein, A. Sanpera, and V. Ahufinger, Ultracold Atoms in Optical Lattices - Simulating quantum manybody systems (Oxford University Press, 2012)

[5] V. Schweikhard et al., Phys. Rev. Lett. 92, 040404 (2004)

[6] N. Cooper, Adv. Phys. 57, 539 (2008)

[7] J. Dalibard, F. Gerbier, G. Juzeliūnas, and P. Öhberg, Rev. Mod. Phys. 83, 1523 (2011)B. Juliá-Díaz et al., Phys. Rev. A 84, 053605 (2011)New J. Phys. 14, 055003 (2012)

[8] Y.-J. Lin et al., Nature 462, 628 (2009)Phys. Rev. Lett. 102, 130401 (2009)

[9] J. Ruseckas, G. Juzeliūnas, P. Öhberg, and M. Fleischhauer, Phys. Rev. Lett. 95, 010404 (2005)

[10] Y. J. Lin, K. Jiménez-García, and I. B. Spielman, Nature 471, 83 (2011)
[11] N. R. Cooper, N. K. Wilkin, and J. M. F. Gunn, Phys. Rev. Lett. 87, 120405 (2001)

[12] N. Read and E. Rezayi, Phys. Rev. B 59, 8084 (1999)

[13] R. B. Laughlin, Phys. Rev. Lett. 50, 1395 (1983)

[14] H.-J. Miesner et al., Phys. Rev. Lett. 82, 2228 (1999)C. J. Myatt et al., ibid. 78, 586 (1997)

[15] E. J. Mueller and T.-L. Ho, Phys. Rev. Lett. 88, 180403 (2002)S. Bargi, J. Christensson, G. M. Kavoulakis, and S. M. Reimann, ibid. 98, 130403 (2007)K. Kasamatsu, M. Tsubota, and M. Ueda, ibid. 91, 150406 (2003)

[16] M. R. Matthews et al., Phys. Rev. Lett. 83, 2498 (1999)V. Schweikhard et al., ibid. 93, 210403 (2004)

[17] E. Ardonne and K. Schoutens, Phys. Rev. Lett. 82, 5096 (1999)E. Ardonne, N. Read, E. Rezayi, and K. Schoutens, Nucl. Phys. B 607, 549 (2001)B. Estienne and B. A. Bernevig, ibid. 857, 185 (2012)

[18] B. I. Halperin, Helv. Phys. Acta 56, 75 (1983)

[19] M. Burrello and A. Trombettoni, Phys. Rev. Lett. 105, 125304 (2010) Phys. Rev. A 84, 043625 (2011)

[20] R. N. Palmer and J. K. Pachos, New J. Phys. 13, 065002 (2011)S. Komineas and N. R. Cooper, Phys. Rev. A 85, 053623 (2012)

[21] D. Yoshioka, B. I. Halperin, and P. A. Lee, Phys. Rev. Lett. 50, 1219 (1983)

[22] F. D. M. Haldane, Phys. Rev. Lett. 55, 2095 (1985)

[23] S. Furukawa and M. Ueda, arXiv:1205.2169 